Research Article

\title{
On Multitracking of First-Order MASs with Adaptive Coupling Strength
}

\author{
Da Huang $\mathbb{D}^{1},{ }^{1}$ Xiaolin Fan, ${ }^{1}$ Zhiyong Yu, ${ }^{2}$ and Haijun Jiang ${ }^{2}$ \\ ${ }^{1}$ Department of Mathematics and Physics, Xinjiang Institute of Engineering, Urumqi 830023, Xinjiang, China \\ ${ }^{2}$ College of Mathematics and System Sciences, Xinjiang University, Urumqi 830046, Xinjiang, China \\ Correspondence should be addressed to Da Huang; xiaoda86op@163.com
}

Received 20 August 2020; Revised 20 October 2020; Accepted 22 October 2020; Published 19 November 2020

Academic Editor: Stefan Balint

Copyright (c) 2020 Da Huang et al. This is an open access article distributed under the Creative Commons Attribution License, which permits unrestricted use, distribution, and reproduction in any medium, provided the original work is properly cited.

The problem of cluster consensus with multiple leaders is called multitracking. In this article, a sort of multitracking of first-order multiagent systems with adaptive coupling strength is studied by the application of adaptive strategy, and the delayed relation between various leaders and clusters is considered. To reach the clustered multitracking goal, a novel pinning-like control protocol with adaptive approach is designed according to the properties of network topology. In addition, the structure of the networked system is a weakly connected digraph. Some conditions are derived to ensure that the nodes in the same cluster reach the consensus via tracking their leader, while leaders will keep a delayed relation with the settled leader node as time goes on to form the required delay consensus.

\section{Introduction}

A multiagent system (MAS) is a networked system consisted of multiple interconnected computerized agents. Consensus is a sort of distributed coordination problem of MASs, and one basic aim for the research is to design reasonable control strategies so that all agents can achieve a common status value. In the system model related with consensus, the agents are required to communicate with each other based on the graph of the networked system so that they can cooperate effectively to finish some desired tasks.

As one of the interdisciplinary research topics related to the field of applied mathematics, control theory, and computer science, consensus problem has attracted much attention of both researchers and engineers thanks to its wide application in the coordination problem of aerial vehicles, satellite clusters, sensor networks, etc. In the past decades, consensus problems have been explored from various aspects in the existing research works [1-14], and during this period, synchronization problems of complex networks, which share similar control strategies with consensus problems, have also been widely investigated [15-34]. The study methods for the two coordination problems are essentially the same.
Cluster consensus, as a sort of extended consensus problems, means that all agents in one cluster achieve the same target status while there exists no consensus for agents among different clusters. As the scales and complexities of complex systems increase, cluster consensus problem exhibits more flexibility in practical applications, and it has a more general concept than the original global consensus. In fact, many multitasking issues need to be solved by partitioning all the nodes into different clusters. It is familiar that the clustered consensus phenomenon is very common in practical circumstances, such as formation of group opinions and multispecies foraging. The case of cluster consensus with multileaders is usually called as multitracking, for which the main aim is to make the reasonable controller design so that the position or velocity status of nodes in each cluster will be consistent with that of the leader.

Over the past decade, the study of coordination control related to clustered structure has caused wide attention, and an amount of research works on clustered networks have been done from various aspects $[2,4-7,9,10$, $13-15,17-22,31-33]$.

In [15], some simple and useful criteria are derived by constructing an effective control scheme and adjusting automatically the adaptive coupling strength. In [17], 
the article investigated the cluster synchronization with time-varying coupling strengths and delayed dynamical systems by applying pinning scheme. Ma et al. [9] investigated the second-order group consensus through pinning control and leader-following approach, and the pinning scheme is established by the structural properties of the graph.

Based on the rationality of the existence of time delays in the coordination system, many study works on clustered networked systems with time delays have been done $[2,15,17,22]$. Ma et al. [2] have proposed the notion of cluster-delay consensus. In [2], the authors find a method to deal with problem on the delayed relations between different clusters through the tracking process of the leader nodes. In real situation, the notion of cluster-delay consensus may imply that the nodes in different clusters should arrive at the same position at different times, which means the possible congestion can be avoided in the MASs.

As we know, the studies on cluster consensus models which considered the possible existence of communication links among the multiple leader nodes are relatively rare.

In real systems, the graph structure may be very complicated and has the group pattern because of the accomplishment of multitasks which have some exact delayed relations of final states. For instance, in the traffic system, to avoid congestion and make roads orderly, time delays can be designed among different sorts of vehicles.

It is familiar that an adaptive controller is a controller that modifies its properties to adapt to changes in the dynamics properties of disturbances, and the adaptive control $([15,16,18,35,36])$ strategy which acts on the coupling strengths of networks is very effective in enhancing the stability of consensus problem. In the study of clustered coordinate control problem, many researchers use adaptive approach to synchronize the complex network or to make the MAS reach consensus.

Through the above observations and the advantages of adaptive control, an issue arises naturally: How can this sort of multitracking problem be solved if the adaptive couplings and the adaptive control method are involved, and what sort of control strategy can be suitably designed for reaching the consensus? For solving this problem, we establish multitracking models with adaptive coupling strength, and this sort of cluster consensus will be realized by designing suitable adaptive control strategies.

Specifically, the novelties of this research are listed as follows:

(i) This article studies a novel multitracking model with adaptive coupling strength, and the delayed relation among the states of agents in different clusters is embodied through the tracking process for the leading subsystem

(ii) Under the clustered digraph, a new pinning-like control strategy combined with adaptive approach is designed

(iii) Compared with similar problems on cluster consensus, two kinds of time delays and two sorts of adaptive coupling strengths are considered in this paper

In this paper, the intrinsic dynamics of all followers and leader nodes satisfy a Lipschitz-like condition. Lyapunov method, matrix theory, and graph theory are used for deriving the results which can solve the multitracking problem.

The rest part is organized as follows. Model description and some preliminaries are given in Section 2. Section 3 includes the main results. Section 4 gives the numerical simulations to verify the theoretical results, and Section 5 has made the final conclusions.

Notation: throughout this article, $R$ represents the set of real numbers. Let $R^{n}$ denotes the $n$-dimensional Euclidean space and $R^{M \times N}$ denotes the $M \times N$ real matrices. $O_{N \times N}$ denotes the zero matrix, and $I_{n}$ is the $n$-dimensional identity matrix. For a real matrix $A \in R^{N \times N}$, let $A^{\mathrm{T}}$ be its transpose and denote its symmetric matrix as $A_{s}=\left(A+A^{\mathrm{T}}\right) / 2$, and $\lambda_{2}(A)$ denotes the maximum eigenvalue of $A$. The norm of a vector is denoted by $\|x\|=\left(x^{T} x\right)^{1 / 2}$, for $x \in R^{n}$. For a real symmetric matrix $F$, denote $F<0(F>0)$, if $F$ is negative (positive) definite. For any two nonempty sets $\mathscr{X}$ and $\mathscr{Y}$, $\mathscr{X} \backslash \mathcal{Y}$ denotes the complementary set of $\mathscr{Y}$ respect to $\mathscr{X} . \otimes$ is the Kronecker product.

\section{Model Description}

In this research, the graph structure of a MAS is denoted by a digraph $\mathscr{B}=\{\mathbb{V}, \mathbb{E}, \mathbb{A}\}$, where the vertex set $\mathbb{V}=\left\{v_{1}, v_{2}, \ldots, v_{N}\right\}$ denotes the agents, $\mathbb{E} \subseteq \mathbb{V} \times \mathbb{V}$ is the edge set, and $\mathbb{A}=\left[a_{i j}\right]_{N}$ is the weighted adjacency matrix which denotes the linking structure of the MAS. A directed edge of $\mathscr{B}$ denoted by $\left(v_{i}, v_{j}\right)$ means that there is a directed information link from $v_{i}$ to $v_{j}$, which can be understood as $v_{j}$ can receive information from $v_{i}$. The elements of $\mathbb{A}$ are defined as follows: $a_{i j}>0$, if $\left(v_{j}, v_{i}\right) \in \mathbb{E} ; a_{i j}=0$, if $\left(v_{j}, v_{i}\right) \notin \mathbb{E}$.

The digraph is supposed to be simple [37] and weakly connected. The in-degree of node $v_{i}$ is defined as $\operatorname{deg}_{i n}\left(v_{i}\right)=\sum_{j=1, j \neq i}^{N} a_{i j}$. The Laplacian matrix of $\mathscr{B}$ is denoted by $L=\left(l_{i j}\right) \in R^{N \times N}$ and is defined as $l_{i j}=-a_{i j}, i \neq j$. $l_{i i}=\sum_{j=1, j \neq i}^{N} a_{i j}$, which ensures that $\sum_{j=1}^{N} l_{i j}=0$.

Let us consider a first-order multiagent network consisting of $N$ followers and $m$ leader nodes. The dynamics of the follower with time-varying delay is modeled by

$$
\begin{aligned}
\dot{x}_{i}(t)= & f\left(x_{i}(t), x_{i}(t-\varsigma(t))\right)+c(t) \sum_{j \neq i} a_{i j}\left(x_{j}(t)-x_{i}(t)\right) \\
& +u_{i}(t), \quad i=1,2, \ldots, N,
\end{aligned}
$$

where $x_{i}(t) \in R^{n}$ is the state of the $i$ th node, $u_{i}(t) \in R^{n}$ is the control input, and $f\left(x_{i}(t), x_{i}(t-\varsigma(t))\right) \in R^{n}$ is the intrinsic nonlinear dynamics of the ith agent. $c(t)>0$ is the timevarying coupling strength. $\varsigma(t)>0$ is the inherent timevarying delay.

Suppose the networked system has $m$ clusters with $2 \leq m<N$, and each cluster has one leader. Let $V_{r}$ be the 
node set of the $r$ th cluster, thus $\mathbb{V}=\left\{v_{1}, v_{2}, \ldots, v_{N}\right\}=$ $\mathbb{V}_{1} \cup \mathbb{V}_{2} \cup \ldots \cup \mathbb{V}_{m} \quad$ and $\quad \mathbb{V}_{r} \cap \mathbb{V}_{s}=\varnothing \quad(r, s=1,2, \ldots$, $m ; r \neq s)$. Let $\widehat{i}$ be the subscript of the cluster that the $i$ th vertex belongs, i.e., $v_{i} \in \mathbb{V}_{\hat{i}}$. Let the leader set be $\mathbb{V}^{*}=\left\{v_{1}^{*}, v_{2}^{*}, \ldots, v_{m}^{*}\right\}$. The followers of the $r$ th leader are the nodes in $\mathbb{V}_{r}, r \in\{1,2, \ldots, m\}$. Let $\widetilde{\mathbb{V}}_{r} \subseteq \mathbb{V}_{r}$ be subset of nodes which can receive information from other clusters, i.e., for any vertex $v_{i} \in \widetilde{\mathbb{V}}_{r}$, there exists at least one node $v_{j} \in \mathbb{V} \backslash \mathbb{V}_{p}$ such that $a_{i j} \neq 0$.

The leaders of clusters for system (1) are described by the following equation:

$$
\begin{array}{r}
\dot{h}_{w}(t)=f\left(h_{w}(t), h_{w}(t-\varsigma(t))\right)-\gamma_{w}\left(h_{w}(t)-h_{1}\left(t-\varsigma_{w}\right)\right), \\
w=1,2, \ldots, m,
\end{array}
$$

where $h_{w}(t)$ is the state of $v_{k}^{*}, \gamma_{w}>0$ is the coupling coefficient between $v_{w}^{*}$ and $v_{1}^{*}$, and $\varsigma_{w}$ is the time delay between the $v_{w}^{*}$ and $v_{1}^{*}, \varsigma_{1}=0$ and $\varsigma_{w}>0, w=2, \ldots, m$.

Remark 1. We can deduce from (2) that the linking structure of the term $-\gamma_{w}\left(h_{w}(t)-h_{1}\left(t-\varsigma_{w}\right)\right)$ implies a star coupled digraph, which has $v_{1}^{*}$ as its center vertex and $v_{w}^{*}$ as its leaf vertex. The multitracking model can be interpreted as adding pinning communication links between agents of the star subnetwork and the pinned nodes of the clustered subnetwork (see Figure 1)

The main goal of the research is to impose suitable control effects $u_{i}(t)$ on subsystem (1) such that it can keep pace with the subsystems (2) with pinning-like adaptive approach. In the meantime, subnetwork (2) is designed to reach the desired delayed consensus to be defined later. For

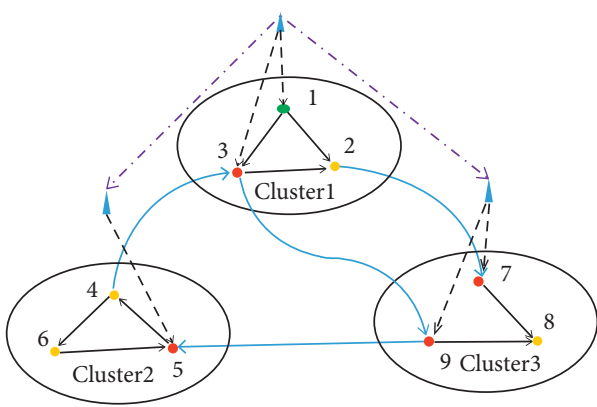

FIgURE 1: The graph of a multitracking model. The $k$ th leader is denoted by triangle dot. The $i$ th agent is labelled with $i$ and denoted by circle dot, $i=1,2, \ldots, 12 ; k=1,2,3$.

getting the main conclusions, the following definition and assumptions are necessary.

Definition 1. The MAS with delayed inherent dynamics (1) and (2) is said to reach the delayed cluster consensus, if the solutions of (1) and (2) satisfy $\lim _{t \longrightarrow \infty}\left\|x_{i}(t)-h_{i}(t)\right\|=0$ and $\lim _{t \rightarrow \infty}\left\|h_{w}(t)-h_{1}\left(t-\varsigma_{w}\right)\right\|=0$.

Remark 2. One can see from the dynamics that the movement of $v_{1}^{*}$ is determined by its own dynamic behavior. In fact, the MAS with (1) and (2) can be interpreted as an entire system consisting of two subsystems. The entire network is designed to achieve the delayed cluster consensus via applying adaptive control strategies.

Assumption 1. There exist two positive constants $\theta$ and $\eta$, such that

$$
\begin{aligned}
& (\phi(t)-\varphi(t))^{T}(f(\phi(t), \phi(t-\varsigma(t)))-f(\varphi(t), \varphi(t-\varsigma(t)))) \\
& \quad \leq \theta(\phi(t)-\varphi(t))^{T}(\phi(t)-\varphi(t))+\eta(\phi(t-\varsigma(t))-\varphi(t-\varsigma(t)))^{T}(\phi(t-\varsigma(t))-\varphi(t-\varsigma(t))),
\end{aligned}
$$

for any $\varphi(t), \phi(t) \in R^{n}$, where $\varsigma(t)$ is the time-varying delay of intrinsic dynamic.

Assumption 2. The inequalities $0<\varsigma(t) \leq \varsigma$ and $0<\dot{\varsigma}(t)$ $\leq \epsilon<1$ hold for the time-varying delay $\varsigma(t)$, where $\varsigma$ and $\epsilon$ are constants.

Lemma 1 (see [9]). Based on the notations of Section 2, if node $v_{i} \in \mathbb{V}_{\widehat{i}} \backslash \widetilde{\mathbb{V}}_{\widehat{i}}$, then $\sum_{j=1}^{N} l_{i j} h_{\widehat{j}}\left(t-\varsigma_{\widehat{j}}\right)=0$.

Lemma 2 (see [38]). For matrices $\mathscr{A}, \mathscr{B}, \mathscr{C}$, and $\mathscr{D}$ with suitable dimensions, the following equations hold:

(i) $(\mathscr{A}+\mathscr{B}) \otimes \mathscr{C}=\mathscr{A} \otimes \mathscr{C}+\mathscr{B} \otimes \mathscr{C}$

(ii) $(\mathscr{A} \otimes \mathscr{B})^{T}=\mathscr{A}^{T} \otimes \mathscr{B}^{T}$

Lemma 3 (see [11]). Let $\vartheta(\cdot):\left[t_{0}-\varsigma,+\infty\right) \longrightarrow[0,+\infty)$ be a continuous function and $\vartheta(t) \leq-c \vartheta(t)+d \bar{\vartheta}(t)$ holds for $t \geq t_{0}$, where $\bar{\vartheta}(t)=s u p_{-\varsigma \leq \zeta \leq 0}(\vartheta(t+\zeta))$. If $c>d>0$, then one has

$$
\vartheta(t) \leq \bar{\vartheta}\left(t_{0}\right) \exp \left\{-\epsilon\left(t-t_{0}\right)\right\}, \quad t \geq t_{0},
$$

where $\epsilon>0$ satisfies the equation: $\epsilon-c+\operatorname{dexp}\{\epsilon \varsigma\}=0$.

\section{Main Results}

Since $l_{i j}=-a_{i j}, i \neq j$, and $l_{i i}=\sum_{j=1, j \neq i}^{N} a_{i j}$, (1) can be rewritten by

$$
\begin{array}{r}
\dot{x}_{i}(t)=f\left(x_{i}(t), x_{i}(t-\varsigma(t))\right)-c(t) \sum_{v_{j} \in \mathbb{V}} l_{i j} x_{j}(t)+u_{i}(t), \\
v_{i} \in \mathbb{V} .
\end{array}
$$

The consensus error in each cluster is as follows:

$$
\xi_{i}(t)=x_{i}(t)-h_{\widehat{i}}(t) .
$$

Then the error system with (1) and (2) can be obtained: 


$$
\begin{aligned}
\dot{\xi}_{i}(t)= & f\left(x_{i}(t), x_{i}(t-\varsigma(t))\right)-f\left(h_{\widehat{i}}(t), h_{\widehat{i}}(t-\varsigma(t))\right) \\
& -c(t) \sum_{j=1}^{N} l_{i j} \xi_{j}(t)-c(t) \sum_{j=1}^{N} l_{i j} h_{\widehat{j}}(t) \\
& +\gamma_{\widehat{i}}\left(h_{\widehat{i}}(t)-h_{1}\left(t-\varsigma_{\widehat{i}}\right)\right)+u_{i}(t) .
\end{aligned}
$$

By the network structure and Lemma 1, the control input $u_{i}(t)$ can be constructed as follows:

$$
u_{i}(t)= \begin{cases}-\gamma_{\widehat{i}}\left(h_{\widehat{i}}(t)-h_{1}\left(t-\varsigma_{\widehat{i}}\right)\right)+c(t) \sum_{j=1}^{N} l_{i j} h_{\widehat{j}}(t)-c(t) \mathrm{d}_{i} \xi_{i}(t), & v_{i} \in \widetilde{\mathbb{V}}_{\widehat{i}} \\ -\gamma_{\widehat{i}}\left(h_{\widehat{i}}(t)-h_{1}\left(t-\varsigma_{\widehat{i}}\right)\right)-c(t) d_{i} \xi_{i}(t), & v_{i} \in \mathbb{V}_{\widehat{i}} \backslash \widetilde{\mathbb{V}}_{\widehat{i}} \wedge \operatorname{deg}\left(v_{i}\right)_{i n}=0 \\ -\gamma_{\widehat{i}}\left(h_{\widehat{i}}(t)-h_{1}\left(t-\varsigma_{\widehat{i}}\right)\right), & \text { otherwise, }\end{cases}
$$

where $c(t)>0$ is the adaptive coupling strength, $d_{i}>0$ is the feedback control gain, and the adaptive updating law is as follows:

$$
\dot{c}(t)=\alpha \sum_{i=1}^{N} \xi_{i}^{\mathrm{T}}(t) \xi_{i}(t)
$$

where $\alpha>0$ is the adaptive gain.

For simplicity, the nodes in $\mathbb{V}_{-}$can be classified as $\mathbb{V}_{1}^{i}=\tilde{\mathbb{V}}_{\hat{i}}, \mathbb{V}_{2}^{i}=\left\{v_{i} \mid v_{i} \in \mathbb{V}_{\hat{i}} \backslash \widetilde{\mathbb{V}}_{\hat{i}}, \operatorname{deg}\left(v_{i}\right)_{i n}=0\right\}$, and $\mathbb{V}_{3}^{i}=\mathbb{V}_{\hat{i}} \backslash$ $\left(\mathbb{V}_{1}^{i} \cup \mathbb{V}_{2}^{i}\right)$. Let $D=\operatorname{diag}\left\{d_{1}^{i}, d_{2}, \ldots, d_{N}\right\}$ with $d_{i}>0$ for $v_{i} \in\left(\mathbb{V}_{1}^{i} \cup \mathbb{V}_{2}^{i}\right)$ and $d_{i}=0$ for $v_{i} \in \mathbb{V}_{3}^{i}$.
Remark 3. By the form of controller (8), one can see that in the $k$ th cluster $(k=2,3, \ldots, m)$, each of the three types of nodes has been controlled by individual scheme, i.e., to the nodes $v_{i} \in\left(\mathbb{V}_{1}^{i} \cup \mathbb{V}_{2}^{i}\right)$, the first term is used for counteracting the interaction between the leaders $v_{k}^{*}$ and $v_{1}^{*}$, while the third term $-c(t) d_{i} \xi_{i}(t)$ is a feedback term exert on the $i$ th node, and it is applied to make the nodes reach the consensus intra one cluster. The second term applied to the nodes in $v_{i} \in \widetilde{\mathbb{V}}_{\hat{i}}$ is used to balance the interactions among clusters.

By (7) and (8), the error system with adaptive coupling strength has the following description:

$$
\dot{\xi}_{i}(t)=f\left(x_{i}(t), x_{i}(t-\varsigma(t))\right)-f\left(h_{\widehat{i}}(t), h_{\widehat{i}}(t-\varsigma(t))\right)-c(t) \sum_{j=1}^{N} l_{i j} \xi_{j}(t)-c(t) d_{i} \xi_{i}(t), \quad v_{i} \in \mathbb{V}_{\hat{i}}
$$

Thus one can acquire the following theorem.

Theorem 1. Based on Assumptions 1 and 2, if the following conditions hold:

(i) $\left(\theta-\beta c^{*}+\eta /(1-\varepsilon)\right)<0$ and $\left(\beta I_{N}-D-L\right)$ is negative definite

(ii) $\tilde{\lambda}<-a_{2}$
Then systems (1) and (2) with protocol (8) and adaptive updating law (9) can solve the multitracking problem, i.e., the delayed cluster consensus can be achieved.

Proof. Consider the following Lyapunov functional candidate:

$$
\mathscr{V}(t)=\frac{1}{2} \sum_{i=1}^{N} \xi_{i}^{\mathrm{T}}(t) \xi_{i}(t)+\frac{\eta}{(1-\epsilon)} \sum_{i=1}^{N} \int_{t-\varsigma(t)}^{t} \xi_{i}^{\mathrm{T}}(s) \xi_{i}(s) \mathrm{d} s+\frac{\beta\left[c(t)-c^{*}\right]^{2}}{2 \alpha},
$$

where $\beta$ and $c^{*}$ are positive constants. 
Denote $\xi(t)=\left(\xi_{1}^{\mathrm{T}}(t), \xi_{2}^{\mathrm{T}}(t), \ldots, \xi_{N}^{\mathrm{T}}(t)\right)^{\mathrm{T}}$, then under Assumptions 1 and 2, by Lemma 1, the time derivative of $\mathscr{V}(t)$ along the trajectories of (10) can be derived as follows:

$$
\begin{aligned}
& \dot{\mathscr{V}}(t)=\sum_{i=1}^{N} \xi_{i}^{\mathrm{T}}(t)\left[f\left(x_{i}(t), x_{i}(t-\varsigma(t))\right)-f\left(h_{\widehat{i}}(t), h_{\widehat{i}}(t-\varsigma(t))\right)\right] \\
& -c(t) \sum_{i=1}^{N} \sum_{j=1}^{N} l_{i j} \xi_{i}^{\mathrm{T}}(t) \xi_{j}(t)-c(t) \sum_{v_{i} \in \widetilde{V}_{\hat{i}}} d_{i} \xi_{i}^{\mathrm{T}}(t) \xi_{i}(t)+\frac{\eta}{(1-\epsilon)} \sum_{i=1}^{N} \xi_{i}^{\mathrm{T}}(t) \xi_{i}(t) \\
& -\frac{(1-\dot{\zeta}(t) \eta)}{(1-\epsilon)} \sum_{i=1}^{N}\left(\xi_{i}^{\mathrm{T}}(t-\varsigma(t)) \xi_{i}(t-\varsigma(t))\right)+\beta\left(c(t)-c^{*}\right) \dot{\bar{c}}(t) \\
& \leq \theta \sum_{i=1}^{N} \xi_{i}^{\mathrm{T}}(t) \xi_{i}(t)+\eta \sum_{i=1}^{N} \xi_{i}^{\mathrm{T}}(t-\varsigma(t)) \xi_{i}(t-\varsigma(t)) \\
& -c(t) \xi^{\mathrm{T}}(t)\left(L \otimes I_{n}\right) \xi(t)-c(t) \sum_{v_{i} \in \widetilde{V}_{\hat{i}}} d_{i} \xi_{i}^{\mathrm{T}}(t) \xi_{i}(t)+\frac{\eta}{(1-\epsilon)} \sum_{i=1}^{N} \xi_{i}^{\mathrm{T}}(t) \xi_{i}(t) \\
& -\frac{(1-\dot{\zeta}(t)) \eta}{(1-\epsilon)} \sum_{i=1}^{N}\left(\xi_{i}^{\mathrm{T}}(t-\varsigma(t)) \xi_{i}(t-\varsigma(t))\right) \\
& +\beta\left[c(t)-c^{*}\right] \sum_{i=1}^{N} d_{i} \xi_{i}^{\mathrm{T}}(t) \xi_{i}(t) \\
& =\theta \xi^{\mathrm{T}}(t) \xi(t)+\eta \xi^{\mathrm{T}}(t-\varsigma(t)) \xi(t-\varsigma(t))-c(t) \xi^{\mathrm{T}}(t)\left(L \otimes I_{n}\right) \xi(t) \\
& +\frac{\eta}{(1-\epsilon)} \xi^{\mathrm{T}}(t) \xi(t)-\frac{(1-\dot{\zeta}(t)) \eta}{(1-\epsilon)}\left(\xi^{\mathrm{T}}(t-\varsigma(t)) \xi(t-\varsigma(t))\right) \\
& +\beta c(t) \xi^{\mathrm{T}}(t)\left(D \otimes I_{n}\right) \xi(t)-\beta c^{*} \xi^{\mathrm{T}}(t)\left(I_{N} \otimes I_{n}\right) \xi(t) \\
& -c(t) \xi^{\mathrm{T}}(t)\left(D \otimes I_{n}\right) \xi(t) \\
& \leq \theta \xi^{\mathrm{T}}(t) \xi(t)-c(t) \xi^{\mathrm{T}}(t)\left(L \otimes I_{n}\right) \xi(t)+\frac{\eta}{(1-\varepsilon)} \xi^{\mathrm{T}}(t) \xi(t)-\beta c^{*} \xi^{\mathrm{T}}(t)\left(I_{N} \otimes I_{n}\right) \xi(t) \\
& +\beta c(t) \xi^{\mathrm{T}}(t)\left(I_{N} \otimes I_{n}\right) \xi(t)-c(t) \xi^{\mathrm{T}}(t)\left(D \otimes I_{n}\right) \xi(t) \\
& \leq \xi^{\mathrm{T}}(t)\left(\left(\theta-\beta c^{*}+\frac{\eta}{(1-\epsilon)}\right) I_{N} \otimes I_{n}+c(t)\left(\beta I_{N}-D-L\right) \otimes I_{n}\right) \xi(t) .
\end{aligned}
$$

One can acquire that $\left(\beta I_{N}-D-Q\right)$ is negative definite for an appropriately small $\beta$ and a sufficiently large $c^{*}$ can ensure $\left(\theta-\beta c^{*}+\eta /(1-\epsilon)\right)<0$ holds, therefore, $\mathscr{V}(t) \leq 0$, which further implies that $\lim _{t \rightarrow \infty}\left\|x_{i}(t)-h-(t)\right\|=0$, $(i=1,2, \ldots, N)$ holds for the solutions of (1) and (2) with any initial values.
On the other aspect, for achieving the delayed consensus among leader nodes, the error for system (2) is denoted by

$$
\bar{\varrho}_{w}(t)=h_{w}(t)-h_{1}\left(t-\varsigma_{w}\right) .
$$

Then the error system is denoted by 


$$
\dot{\bar{\varrho}}_{w}(t)=f\left(h_{w}(t), h_{w}(t-\varsigma(t))\right)-f\left(h_{1}\left(t-\varsigma_{w}\right), h_{1}\left(t-\varsigma_{w}-\varsigma(t)\right)\right)-\gamma_{w} \bar{\varrho}_{w}(t), \quad w=2, \ldots, m \text {. }
$$

The Lyapunov function candidate is established as follows:

$$
\mathscr{V}^{*}(t)=\frac{1}{2} \sum_{w=2}^{m} \bar{\varrho}_{w}^{\mathrm{T}}(t) \bar{\varrho}_{w}(t) .
$$

$$
\begin{aligned}
\dot{\mathscr{V}}^{*}(t) & =\sum_{w=2}^{m} \varrho_{w}^{\mathrm{T}}(t)\left[f\left(h_{w}(t), h_{w}(t-\varsigma(t))\right)-f\left(h_{1}\left(t-\varsigma_{w}\right), h_{1}(t-\varsigma(t))-\varsigma_{w}\right)\right] \\
-\sum_{w=2}^{m} \gamma_{w} \varrho_{w}^{\mathrm{T}}(t) \varrho_{w}(t) & \leq a_{1} \sum_{w=2}^{m} \varrho_{k}^{\mathrm{T}}(t) \varrho_{k}(t)-\sum_{w=2}^{m} \gamma_{w} \varrho_{w}^{\mathrm{T}}(t) \varrho_{w}(t)+a_{2} \sum_{w=2}^{m}\left(\varrho_{w}(t-\varsigma(t))\right)^{\mathrm{T}} \varrho_{w}(t-\varsigma(t)) \\
& \leq \varrho^{* \mathrm{~T}}(t)\left[\left(a_{1} I_{N}-\Theta\right) \otimes I_{n}\right] \varrho^{*}(t)+2 a_{2} \sup _{0 \leq \varsigma(t) \leq \varsigma}\left(\mathscr{V}^{*}(t-\varsigma(t))\right) \\
& \leq 2 \tilde{\lambda} \mathscr{V}^{*}(t)+2 a_{2} \sup _{0 \leq \varsigma(t) \leq \varsigma}\left(\mathscr{V}^{*}(t-\varsigma(t))\right),
\end{aligned}
$$

where $\tilde{\lambda}=\lambda_{\max }\left(a_{1} I_{N}-\Theta\right)$ and $\Theta=\operatorname{diag}\left(\alpha_{2}, \ldots, \alpha_{m}\right)$. Therefore, by Lemma 3 and the condition $\widetilde{\lambda}<-a_{2}$, we have

$$
\begin{aligned}
\mathscr{V}^{*}(t) \leq \sup _{0 \leq \varsigma(0) \leq \varsigma}\left(\mathscr{V}^{*}(-\varsigma(0))\right) \exp \{-\epsilon t\}, \\
\quad \text { where } \epsilon \text { satisfies } \epsilon+2 \widetilde{\lambda}+2 a_{2} \exp \{\epsilon \varsigma\}=0 \text { and } \epsilon>0 .
\end{aligned}
$$

Then one can derive that the delayed consensus among leaders can be achieved in this situation, and therefore, the delayed cluster consensus can be reached under controller (8); furthermore, the uncertain parameters $c(t)$ adapt itself to some certain values, i.e., $c(t) \longrightarrow c^{*}$; thus, the multitracking problem under the adaptive approach is solved, and this completes the proof.

Remark 4. In view of subsystem (2), if $\gamma_{w}$ is time varying and it is described by the form $\gamma_{w}(t)=p_{w} q(t)$, in which $q(t)$ is the coupling strength and $p_{w}$ is the corresponding coupling weights. Set the adaptive updating law as the form: $\dot{q}(t)=\delta \sum_{w=2}^{m} p_{w} \varrho_{w}^{\mathrm{T}}(t) \varrho_{w}(t)$, where $\delta>0$ is the adaptive gain, and thus the second theorem can be obtained.

Theorem 2. Suppose Assumptions 1 and 2 hold, based on Remark 4, if $q(t)$ satisfies the following updating law:

$$
\dot{q}(t)=\delta \sum_{w=2}^{m} p_{w} \varrho_{w}^{\mathrm{T}}(t) \varrho_{w}(t),
$$

and if the following conditions hold:

(i) $\left(\theta-\beta c^{*}+\eta /(1-\epsilon)\right)<0$ and $\left(\beta I_{N}-D-L\right)$ is negative definite

(ii) $\left(\left(a_{1}+\rho /(1-\varepsilon)\right) I_{m-1}-q^{*} \Xi\right)$ is negative definite

Then systems (1) and (2) under controller (8) with adaptive updating law (9) can solve the multitracking problem.

Proof. The proof of the first part is similar to that of Theorem 1 , and to the leading system, we condiser the following Lyapunov functional candidate:

$$
\begin{aligned}
\mathscr{V}^{*}(t)= & \frac{1}{2} \sum_{w=2}^{m} \varrho_{w}^{\mathrm{T}}(t) \varrho_{w}(t)+\frac{1}{2 \delta}\left(q(t)-q^{*}\right)^{2} \\
& +\frac{\rho}{(1-\varepsilon)} \sum_{i=2}^{m} \int_{t-\tau(t)}^{t} \varrho_{i}^{\mathrm{T}}(s) \varrho_{i}(s) \mathrm{d} s,
\end{aligned}
$$

where the constant $q^{*}$ is positive. Denote $\varrho^{*}(t)=\left(\varrho_{2}^{\mathrm{T}}(t), \ldots, \varrho_{m}^{\mathrm{T}}(t)\right)^{\mathrm{T}}, \Xi=\operatorname{diag}\left(q_{2}, q_{3}, \ldots, q_{m}\right), \quad$ and choose $\rho \geq a_{2}$, and then we have 


$$
\begin{aligned}
\dot{\mathscr{V}}^{*}(t) \leq & a_{1} \sum_{w=2}^{m} \varrho_{w}^{\mathrm{T}}(t) \varrho_{w}(t)+a_{2} \sum_{w=2}^{m}\left(\varrho_{w}(t-\varsigma(t))\right)^{\mathrm{T}} \varrho_{w}(t-\varsigma(t))-\sum_{w=2}^{m} p_{w} q(t) \varrho_{w}^{\mathrm{T}}(t) \varrho_{w}(t) \\
& +\sum_{w=2}^{m} p_{w} q(t) \varrho_{w}^{\mathrm{T}}(t) \varrho_{w}(t)-q^{*} \sum_{w=2}^{m} p_{w} \varrho_{w}^{\mathrm{T}}(t) \varrho_{w}(t)+\frac{\rho}{(1-\varepsilon)} \sum_{w=2}^{m} \varrho_{w}^{\mathrm{T}}(t) \varrho_{w}(t)-\frac{(1-\dot{\zeta}(t)) \rho}{(1-\varepsilon)} \\
\sum_{w=2}^{m}\left(\varrho_{w}(t-\varsigma(t))\right)^{\mathrm{T}} \varrho_{w}(t-\varsigma(t)) \leq & a_{1} \sum_{w=2}^{m} \varrho_{w}^{\mathrm{T}}(t) \varrho_{w}(t)-q^{*} \sum_{w=2}^{m} p_{w} \varrho_{w}^{\mathrm{T}}(t) \varrho_{w}(t) \\
& +\frac{\rho}{(1-\varepsilon)} \sum_{w=2}^{m} \varrho_{w}^{\mathrm{T}}(t) \varrho_{w}(t)=\varrho_{w}^{* \mathrm{~T}}(t)\left[\left(\left(a_{1}+\frac{\rho}{(1-\varepsilon)}\right) I_{m-1}-q^{*} \Xi\right) \otimes I_{n}\right] \varrho_{w}^{*}(t)
\end{aligned}
$$

Some sufficiently large $q^{*}$ can be chosen so that $\left(\left(a_{1}+\right.\right.$ $\left.\rho /(1-\varepsilon)) I_{m-1}-q^{*} \Xi\right)$ is negative definite, then $\mathscr{V}^{*}(t) \leq 0$, therefore, the delayed consensus among leaders can be achieved, and therefore, the multitracking problem can be solved, that is, the delayed cluster consensus can be reached. Further, $c(t) \longrightarrow c^{*}$ and $q(t) \longrightarrow q^{*}$, and the proof is completed.
Remark 5. Since the connected undirected graph does not exist vertex with zero in-degree, the control protocol (8) for the network with undirected graph can be simplified as follows:

$$
u_{i}(t)= \begin{cases}-\gamma_{\hat{i}}(t)\left(h_{\hat{i}}(t)-h_{1}\left(t-\varsigma_{\hat{i}}\right)\right)+c(t) \sum_{j=1}^{N} l_{i j} h_{\hat{j}}(t)-c(t) \mathrm{d}_{i} \xi_{i}(t), & v_{i} \in \widetilde{\mathbb{V}}_{\hat{i}}, \\ -\gamma_{\hat{i}}(t)\left(h_{\hat{i}}(t)-h_{1}\left(t-\varsigma_{\hat{i}}\right)\right), & v_{i} \in \mathbb{V}_{\hat{i}} \widetilde{\mathbb{V}}_{\hat{i}}\end{cases}
$$

Under (21), a corollary similar to the previous theorem can be derived, and it is omitted here.

Remark 6. MAS-related problems have been widely analyzed in many fields, such as robotic systems [39], sensor networks [40], and neural networks [41]. Since different networks may contain the same or similar graph structures, the study of one network may shed light on other related networks. One may consider some classical graph structures of the networks, such as neural network, to study the similarity and do some enlightening works in the future research.

\section{Numerical Examples}

In this part, simulation examples are given to check the correctness of the theorem. Consider a MAS composed of three clusters with 9 nodes, and the corresponding graph is shown in Figure 1.

The nodes are labelled with an ascending sequence from 1 to 9 . The purple arrows describe the pinning control that the $k$ th leader put on the nodes with specific topological properties $(k=1,2,3)$. The blue edges denote the interactions among clusters, and the black edges represent the communication links inside each cluster. Through (8) and Figure 1, we know that the red vertexes (labelled by $3,5,7,9$ ) belong to $V_{1}^{i}$, the yellow vertexes $(2,4,6,8)$ belong to $V_{3}^{i}$, and the green vertex (labelled by 1 ) belongs to $V_{2}^{i}$. It is assumed that the dimensional dynamical system of each agent is onedimensional.

Example 1. The nonlinear function $f$ is described by $f\left(\left(x_{i}(t)\right), x_{i}(t-\varsigma(t))\right)=0.5 \sin \left(x_{i}\right)-0.5 \cos \left(x_{i}(t-\varsigma(t))\right)$, where $x_{i}(t), x_{i}(t-\varsigma(t)) \in R$, and $\varsigma(t)=0.25 \tanh (t)$. The elements $l_{i j}$ of graph Laplacian in (5) are chosen as follows: $l_{21}=-1, l_{22}=2, l_{23}=-1, l_{31}=-1, l_{33}=2, l_{34}=-1, l_{44}=1$, $l_{45}=-1, l_{55}=2, l_{56}=-1, l_{59}=-1, l_{64}=-1, l_{66}=1, l_{71}=-1$, $l_{76}=1, l_{86}=-1, l_{87}=2, l_{88}=-1, l_{93}=-1, l_{99}=1$, and if the subscripts of $l_{i j}$ are not involved above, then set $l_{i j}=0$.

The parameters in the adaptive controller (8) with updating law (9) are selected as follows: $\gamma_{1}=0, \gamma_{2}=2, \gamma_{3}=$ $1 ; \quad \varsigma_{1}=0, \varsigma_{2}=0.5, \varsigma_{3}=1 ; \quad d_{1}=2, d_{3}=1.5, d_{5}=2, d_{7}=1$, $d_{9}=0.5$, and $\alpha=0.05$.

In view of Assumption 1 , let $\beta=0.2$, then $\left(I_{N}-D-\right.$ $L)<0$ holds, set $\theta=0.3, \eta=0.7$, and $\epsilon=0.3$, one has $\left(\theta-c^{*}+\eta /(1-\varepsilon)\right)<0$, choose $a_{1}=0.2, a_{2}=0.1$, and thus, the conditions of Theorem 1 can be satisfied. The states 


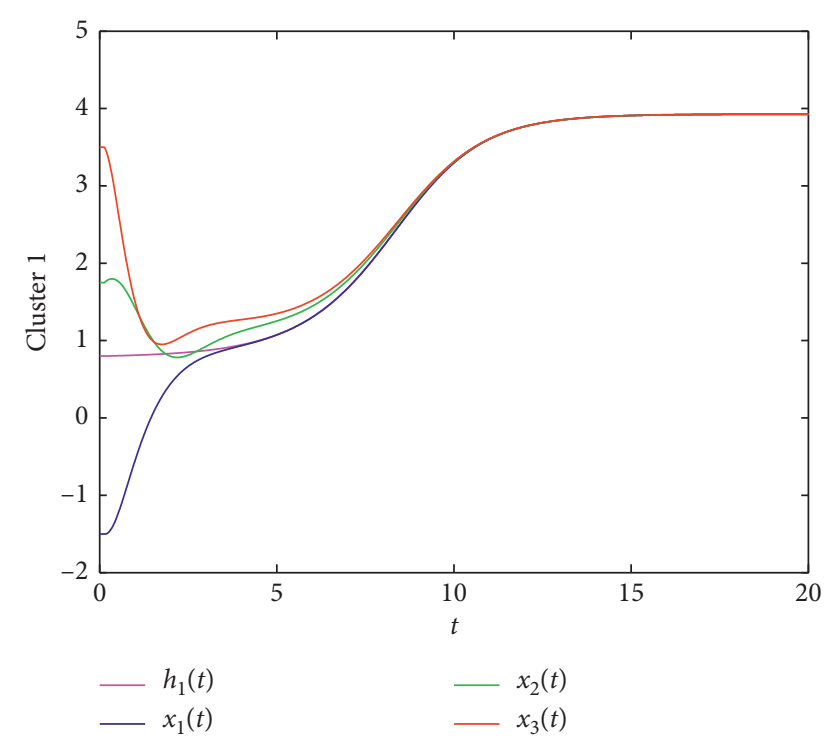

FIgURE 2: State trajectories of cluster 1.

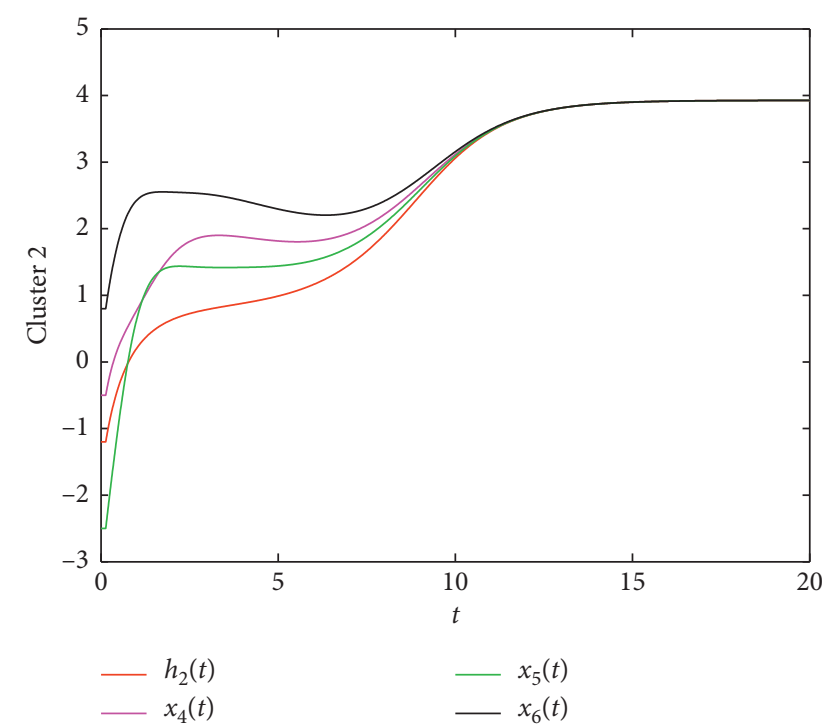

Figure 3: State trajectories of cluster 2.

of all nodes are shown in Figures 2-4, and their initial conditions are as follows: $x_{1}(0)=-1.5, x_{2}$ $(0)=1.75, x_{3}(0)=3.5, x_{4}(0)=-0.5, x_{5}(0)=-2.5, x_{6}(0)=$ $0.8, x_{7}(0)=1.5, \quad x_{8}(0)=-0.8, x_{9}(0)=1.2 ; h_{1}(0)=$ $0.8, h_{2}(0)=-1.2$, and $h_{3}(0)=0.6$. The state changes of leaders are shown in Figure 5. The initial value of $c(t)$ is chosen as $c(0)=0.1$, the orbit of coupling strength $c(t)$ is shown in Figure 6, one can see that $c(t)$ adaptively adjusts with $c(t) \longrightarrow 2$, the two error states of the two subnetworks, i.e., $\xi_{i}(t)$ and $\xi_{w}(t)$ both convergence to zero, and the delayed cluster consensus is indeed achieved.

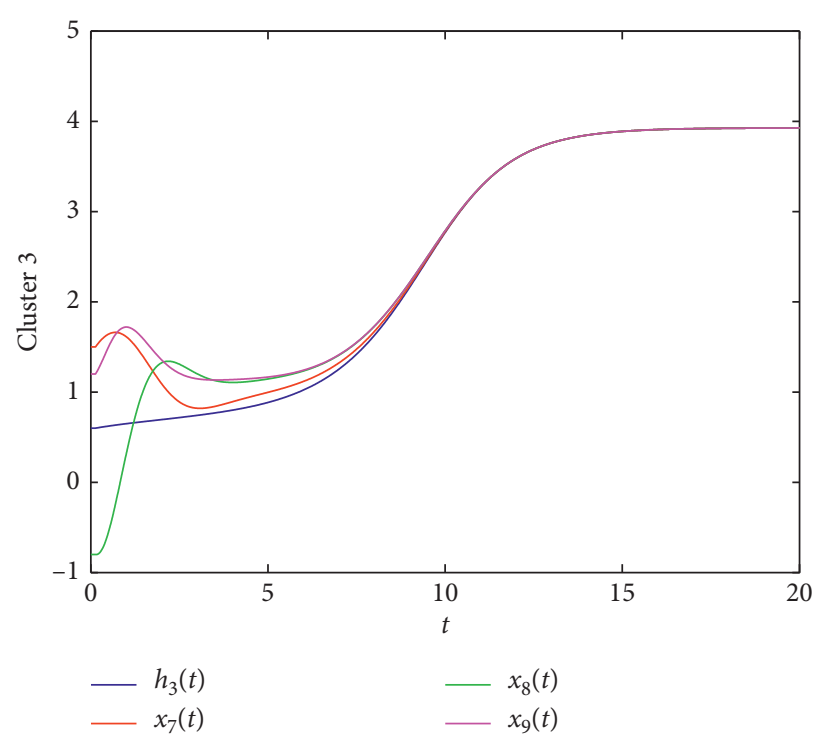

FIGURE 4: State trajectories in cluster 3.

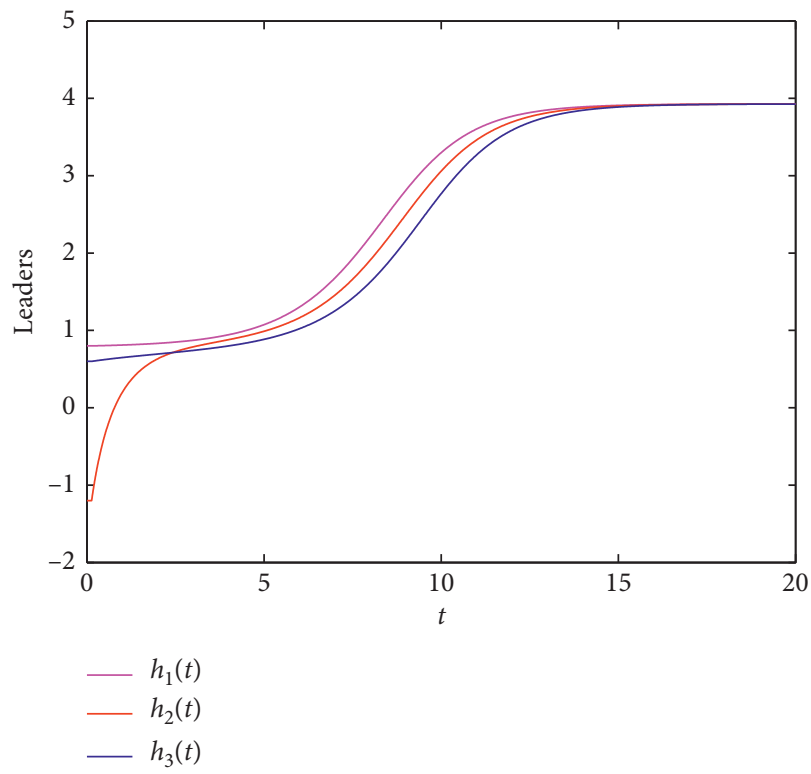

Figure 5: State trajectories of leaders.

Example 2. This example shares the same nonlinear function $f$ with Example 1. Choose the same Laplacian matrix $L=\left(l_{i j}\right)_{9 \times 9}$ with the previous example, and set the initial values as $\quad h_{1}(0)=0.8, h_{2}(0)=-1.2, h_{3}(0)=0.6 ; \gamma_{1}=0, \gamma_{2}(0)=$ $0.02, \gamma_{3}(0)=0.05 ; x_{1}(0)=-1.5, x_{2}(0)=1.75, x_{3}(0)=3.5$, $x_{4}(0)=-0.5, x_{5}(0)=-2.5, x_{6}(0)=0.8, x_{7}(0)=1.5, x_{8} \quad(0)$ $=-0.8, x_{9}(0)=1.2 ; \varsigma(0)=0 ; c(0)=0.1, q(0)=0.01$. Let the parameters in the control protocol be $\varsigma_{1}=0, \varsigma_{2}=0.5, \varsigma_{3}=1$, $d_{1}=2, d_{3}=1.5, \quad d_{5}=2, d_{7}=1, d_{9}=0.5 ; \quad \alpha=0.05, \delta=0.1 ;$ $p_{1}=1, p_{2}=0.5, p_{3}=0.2$; in view of Assumptions 1 and 2 , set 


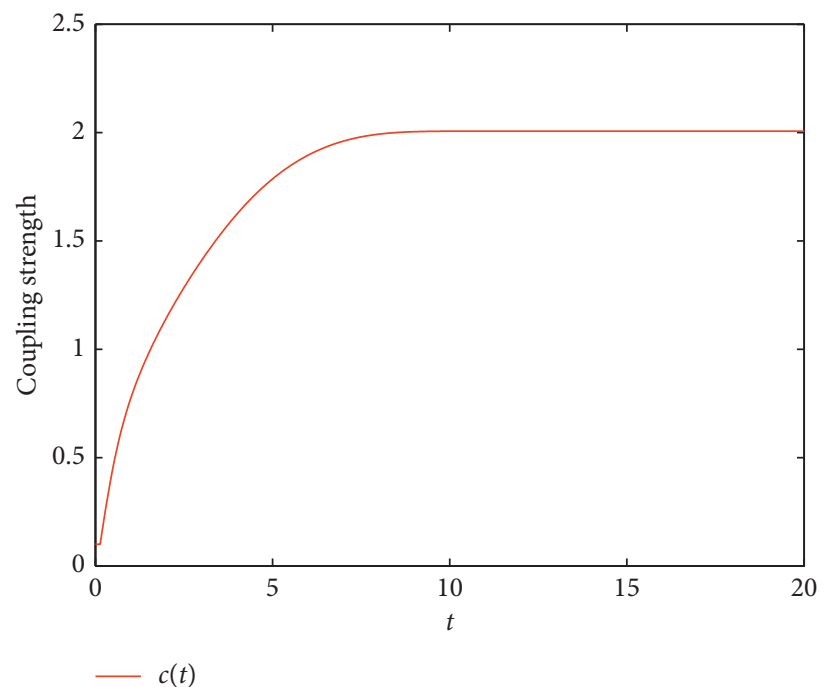

Figure 6: The orbit of adaptive coupling strength $c(t)$.

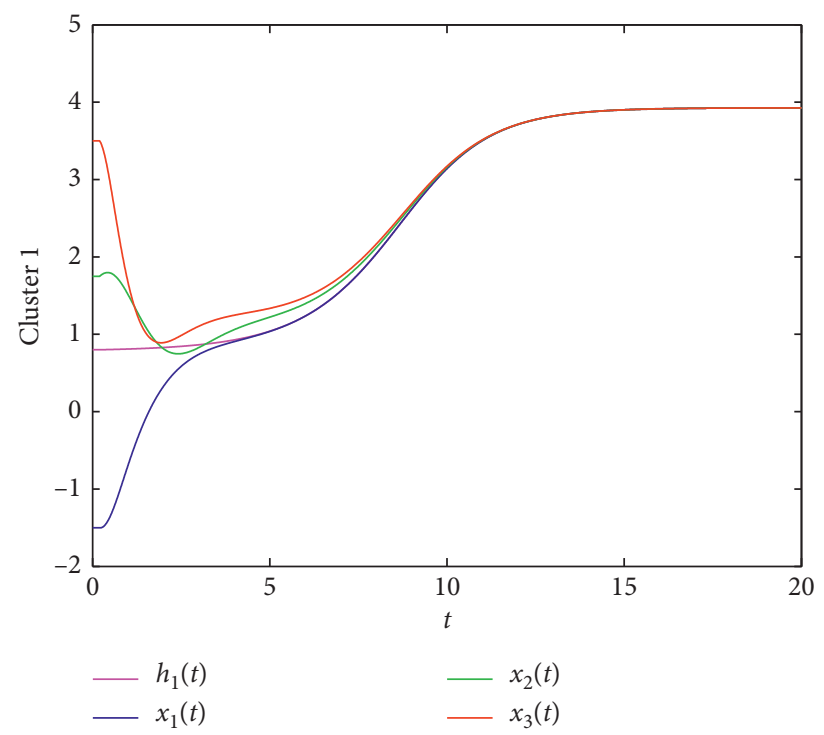

FIgURE 7: The trajectories of state changes in cluster 1.

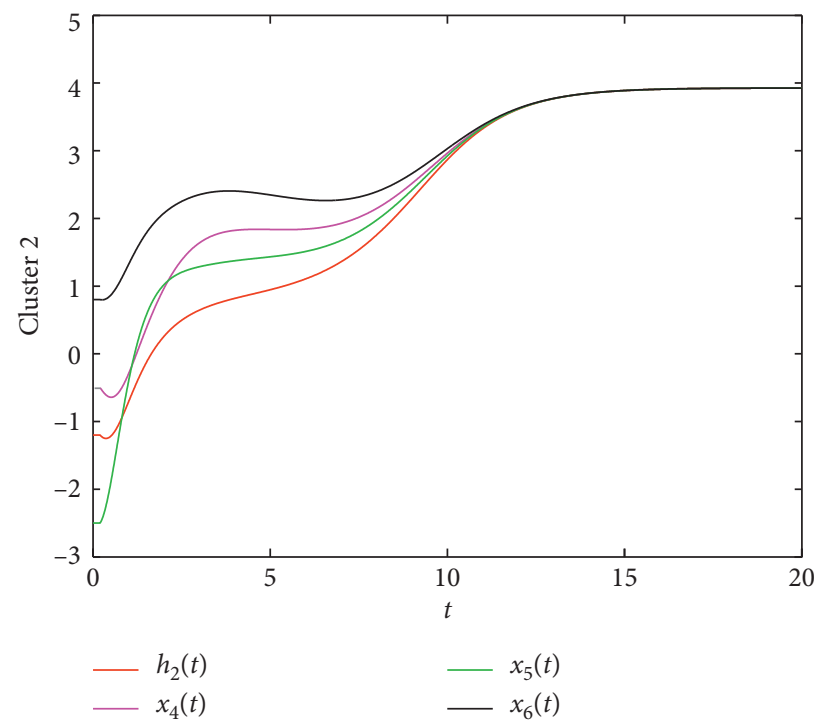

FIgURE 8: The trajectories of state changes in cluster 2. 


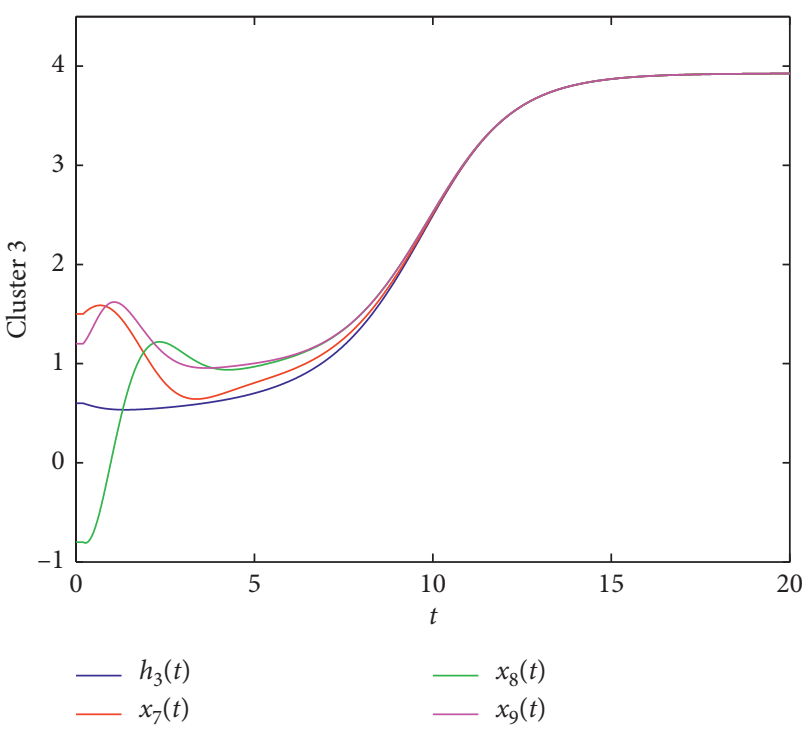

FIgURE 9: The state trajectories in cluster 3.

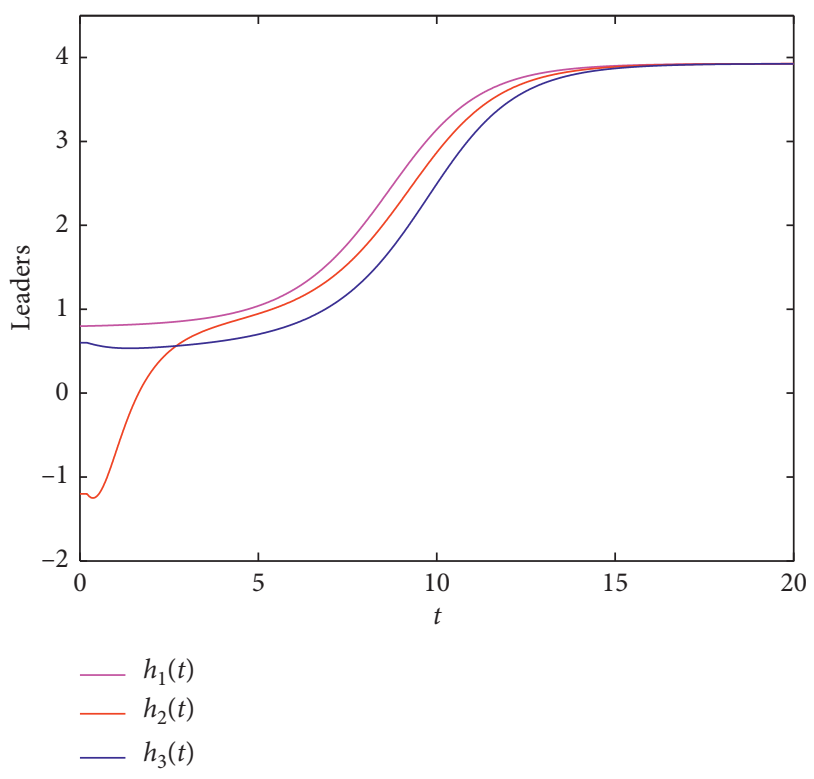

Figure 10: The state trajectories of leaders.

$a_{1}=0.2, a_{2}=0.15, \rho=0.2$, and $\varepsilon=0.25$, and thus, the conditions of Theorem 2 can be satisfied. The description of the state changes of all nodes is shown in Figures 7-10. The change curves of the adaptive couplings $c(t)$ and $\gamma_{k}(t)$ are shown in Figure 11, and one can see that $c(t), \gamma_{k}(t)$ adapt themselves to certain values.

It can be seen that the simulation verifies the effectiveness well, and the newly defined multitracking problem is indeed solved.

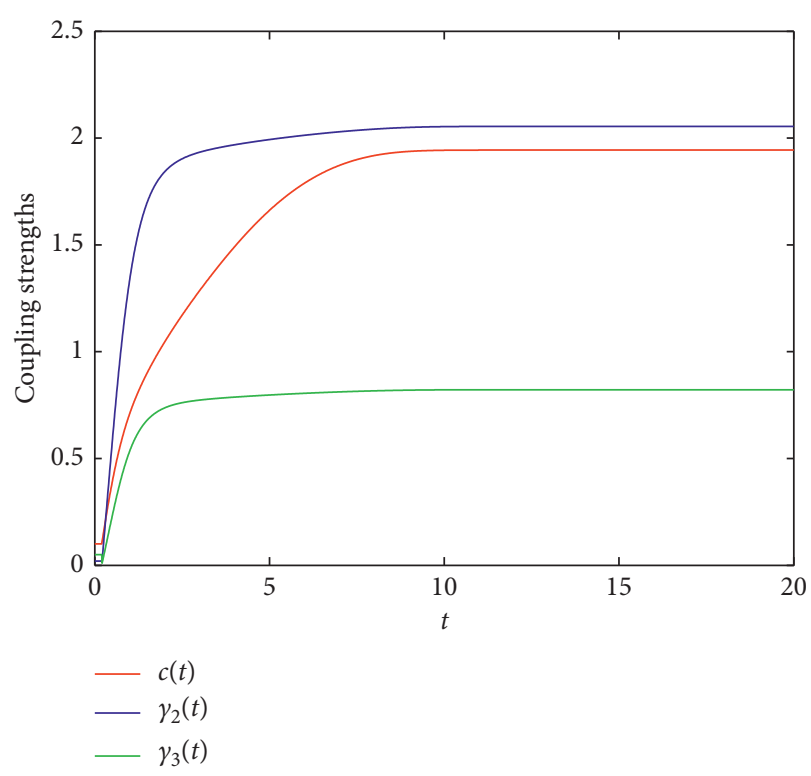

Figure 11: The orbits of $c(t)$ and $\gamma_{k}(t),(k=2,3)$.

\section{Conclusion}

In this work, a type of multitracking problem with adaptive coupling strength named delayed cluster consensus has been investigated. By considering the graph properties of the clustered network, a new pinning-like control scheme with adaptive approach has been designed. Some sufficient criteria for solving the multitracking problem have been obtained. There still exist lots of works on the similar multitracking problem with MAS deserving further study, for example, multitracking issue with higher order system, multitracking with impulsive control, multitracking with switching topology, etc., and some of the problems might be done in one's future research.

\section{Data Availability}

Some or all data, models, or code generated or used during the study are available from the corresponding author by request.

\section{Conflicts of Interest}

The authors declare that they have no conflicts of interest.

\section{Acknowledgments}

The authors are very grateful to the people who provided them valuable help. This work was supported by the Natural Science Foundation of Xinjiang (NSFXJ) (no. 2019D01B10), Youth Doctoral Cultivation Project for Sci-Tech Talents of Xinjiang (2018Q074), National Natural Science Foundation of China (NSFC) (no. 11661077), and Research Fund for the 
Doctoral Program of Xinjiang Institute of Engineering (2018xgy122105).

\section{References}

[1] O. Saber and R. Murray, "Consensus problems in networks of agents with switching topology and time-delays," IEEE Transactions on Automatic Control, vol. 49, no. 9, pp. 15201533, 2004.

[2] Z. Ma, Y. Wang, and X. Li, "Cluster-delay consensus in firstorder multi-agent systems with nonlinear dynamics," Nonlinear Dynamics, vol. 83, no. 3, pp. 1303-1310, 2016.

[3] G. Wen, Z. Duan, W. Ren, and G. Chen, "Distributed consensus of multi-agent systems with general linear node dynamics and intermittent communications," International Journal of Robust and Nonlinear Control, vol. 24, no. 16, pp. 2438-2457, 2014.

[4] Z.-H. Guan, G.-S. Han, J. Li, D.-X. He, and G. Feng, "Impulsive multiconsensus of second-order multiagent networks using sampled position data," IEEE Transactions on Neural Networks and Learning Systems, vol. 26, no. 11, pp. 26782688, 2015.

[5] J. Qin and C. Yu, "Cluster consensus control of generic linear multi-agent systems under directed topology with acyclic partition," Automatica, vol. 49, no. 9, pp. 2898-2905, 2013.

[6] D. Huang, H. Jiang, Z. Yu, and X. Chen, "Cluster-delay consensus in multi-agent systems via pinning leader-following approach with intermittent effect," International Journal of Control, vol. 91, no. 10, pp. 2261-2272, 2018.

[7] J. Yu and L. Wang, "Group consensus of multi-agent systems with directed information exchange," International Journal of Systems Science, vol. 43, no. 2, pp. 334-348, 2012.

[8] F. Chen, Z. Chen, L. Xiang, Z. Liu, and Z. Yuan, "Reaching a consensus via pinning control," Automatica, vol. 45 , no. 5 , pp. 1215-1220, 2009.

[9] Q. Ma, Z. Wang, and G. Miao, "Second-order group consensus for multi-agent systems via pinning leader-following approach," Journal of the Franklin Institute, vol. 351, no. 3, pp. 1288-1300, 2014.

[10] Y. Wang, Z. Ma, and G. Chen, "Avoiding congestion in cluster consensus of the second-order nonlinear multiagent systems," IEEE Transactions on Neural Networks \& Learning Systems, vol. 29, no. 8, pp. 3490-3498, 2018.

[11] Z. Yu, H. Jiang, C. Hu, and X. Fan, "Consensus of secondorder multi-agent systems with delayed nonlinear dynamics and aperiodically intermittent communications," International Journal of Control, vol. 90, no. 5, pp. 909-922, 2017.

[12] G. Wen, Z. Duan, W. Yu, and G. Chen, "Consensus of secondorder multi-agent systems with delayed nonlinear dynamics and intermittent communications," International Journal of Control, vol. 86, no. 2, pp. 322-331, 2013.

[13] G. Wang and Y. Shen, "Second-order cluster consensus of multi-agent dynamical systems with impulsive effects," Communications in Nonlinear Science and Numerical Simulation, vol. 19, no. 9, pp. 3220-3228, 2014.

[14] J. Qin, C. Yu, and B. D. O. Anderson, "On leaderless and leader-following consensus for interacting clusters of secondorder multi-agent systems," Automatica, vol. 74, pp. 214-221, 2016.

[15] C. Hu, J. Yu, H. Jiang, and Z. Teng, "Synchronization of complex community networks with nonidentical nodes and adaptive coupling strength," Physics Letters A, vol. 375, no. 5, pp. 873-879, 2011.
[16] C. Hu and H. Jiang, "Pinning synchronization for directed networks with node balance via adaptive intermittent control," Nonlinear Dynamics, vol. 80, no. 1-2, pp. 295-307, 2015.

[17] S. Wang, H. Yao, S. Zheng, and Y. Xie, "A novel criterion for cluster synchronization of complex dynamical networks with coupling time-varying delays," Communications in Nonlinear Science and Numerical Simulation, vol. 17, no. 7, pp. 29973004, 2012.

[18] L. Shi, H. Zhu, S. Zhong, K. Shi, and J. Cheng, "Cluster synchronization of linearly coupled complex networks via linear and adaptive feedback pinning controls," Nonlinear Dynamics, vol. 88, no. 2, pp. 859-870, 2017.

[19] Z. Wu and X. Fu, "Cluster mixed synchronization via pinning control and adaptive coupling strength in community networks with nonidentical nodes," Communications in Nonlinear Science and Numerical Simulation, vol. 17, no. 4, pp. 1628-1636, 2012.

[20] G. Wang and Y. Shen, "Cluster synchronisation of directed complex dynamical networks with nonidentical nodes via pinning control," International Journal of Systems Science, vol. 44, no. 9, pp. 1577-1586, 2013.

[21] C. Yu, J. Qin, and H. Gao, "Cluster synchronization in directed networks of partial-state coupled linear systems under pinning control," Automatica, vol. 50, no. 9, pp. 2341-2349, 2014.

[22] Z. Wu and X. Fu, "Cluster lag synchronisation in community networks via linear pinning control with local intermittent effect," Physica A, vol. 395, pp. 487-498, 2014.

[23] F. Nian and X. Wang, "Optimal pinning synchronization on directed complex network," Chaos, vol. 21, p. 43131, 2011.

[24] W. Sun, S. Wang, G. Wang, and Y. Wu, "Lag synchronization via pinning control between two coupled networks," Nonlinear Dynamics, vol. 79, no. 4, pp. 2659-2666, 2015.

[25] X. Wu, W. Zheng, and J. Zhou, "Generalized outer synchronization between complex dynamical networks," Chaos, vol. 19, p. 13109, 2009.

[26] W. Yu, G. Chen, and J. Lü, "On pinning synchronization of complex dynamical networks," Automatica, vol. 45, no. 2, pp. 429-435, 2009.

[27] C. Hu, J. Yu, H. Jiang, and Z. Teng, "Pinning synchronization of weighted complex networks with variable delays and adaptive coupling weights," Nonlinear Dynamics, vol. 67, no. 2, pp. 1373-1385, 2011.

[28] R. Lu, W. Yu, J. Lü, and A. Xue, "Synchronization on complex networks of networks," IEEE Transactions on Neural Networks and Learning Systems, vol. 25, no. 11, pp. 2110-2118, 2014.

[29] Q. Song and J. Cao, "On pinning synchronization of directed and undirected complex dynamical networks," IEEE Transactions on Circuits and Systems I: Regular Papers, vol. 57, no. 3, pp. 672-680, 2010.

[30] J. Feng, S. Sun, C. Xu, Y. Zhao, and J. Wang, "The synchronization of general complex dynamical network via pinning control," Nonlinear Dynamics, vol. 67, no. 2, pp. 1623-1633, 2011.

[31] J. Wang, J. Feng, C. Xu, and Y. Zhao, "Cluster synchronization of nonlinearly-coupled complex networks with nonidentical nodes and asymmetrical coupling matrix," Nonlinear Dynamics, vol. 67, no. 2, pp. 1635-1646, 2012.

[32] W. Wu, W. Zhou, and T. Chen, "Cluster synchronization of linearly coupled complex networks under pinning control," IEEE Transactions on Circuits and SystemsI, vol. 56, pp. 829-839, 2009. 
[33] X. B. Lu and B. Z. Qin, "Adaptive cluster synchronization in complex dynamical networks," Physics Letters A, vol. 373, no. 40, pp. 3650-3658, 2009.

[34] Z. Wu, X. Xu, G. Chen, and X. Fu, "Adaptive synchronization and pinning control of colored networks," Chaos, vol. 22, p. 43137, 2012.

[35] H. Zhang, Y. Liu, and Y. Wang, "Observer-based finite-time adaptive fuzzy control for nontriangular nonlinear systems with full-state constraints," IEEE Transactions on Cybernetics, p. 1, 2020.

[36] H. Zhang, Y. Liu, J. Dai, and Y. Wang, "Command filter based adaptive fuzzy finite-time control for a class of uncertain nonlinear systems with hysteresis," IEEE Transactions on Fuzzy Systems, p. 1, 2020.

[37] J. Bondy and U. Murty, Graph Theory, Springer, Berlin, Germany, 2008.

[38] R. Horn and C. Johnson, Matrix Analysis, Cambridge University Press, Cambridge, UK, 2nd edition, 2013.

[39] Y. Pan, P. Du, H. Xue, and H.-K. Lam, "Singularity-free fixedtime fuzzy control for robotic systems with user-defined performance," IEEE Transactions on Fuzzy Systems, p. 1, 2020.

[40] J. Qin, W. Fu, H. Gao, and W. Zheng, "Distributed k-means algorithm and fuzzy c-means algorithm for sensor networks based on multiagent consensus theory," IEEE Transactions on Cybernetics, vol. 47, no. 3, pp. 1-12, 2016.

[41] H. Liang, G. Liu, H. Zhang, and T. Huang, "Neural-networkbased event-triggered adaptive control of nonaffine nonlinear multiagent systems with dynamic uncertainties," IEEE Transactions on Neural Networks and Learning Systems, p. 1, 2020. 